\title{
Experimental studies on structure of airblast spray in crossflow
}

\author{
ANUBHAV SINHA*D and R V RAVIKRISHNA \\ Department of Mechanical Engineering, Indian Institute of Science, Bangalore, India \\ e-mail: er.anubhav@gmail.com
}

MS received 11 November 2017; revised 24 February 2019; accepted 6 March 2019; published online 13 April 2019

\begin{abstract}
The present investigation is focused on experimental studies on the structure of airblast spray in crossflow. Atomizer is at a higher pressure than ambient and the airblast gas is expected to exhibit features generally found in under-expanded gas jets. High-speed images are captured using shadowgraphy technique. Certain wave-like structures observed in the near-nozzle region are attributed to shock-vortex interactions, generally observed in under-expanded gas jets. Proper Orthogonal Decomposition (POD) analysis has been carried out using these images. POD mode shapes clearly show signatures of shock-vortex oscillations and frequencies close to the screeching frequency observed in under-expanded jets. The POD modes are compared for different conditions and the significance of dynamic structures and their temporal behavior is discussed. Finally, a regime map is also proposed to characterize spray behavior in crossflow.
\end{abstract}

Keywords. Spray in crossflow; Proper Orthogonal Decomposition (POD); under-expanded jet; shock-vortex interaction; shock-oscillation; Mach disc; screeching jet; spray regimes.

\section{Introduction}

Spray formation is a critical process for any liquid-fuelled engine. The engine efficiency, emissions and other performance parameters depend strongly on droplet size and their velocities [1]. Injecting spray in crossflow is an attractive configuration for gas turbine engines. Traditionally, liquid fuel is injected as a jet in crossflow of air in gas turbine combustors, afterburners, ramjets and scramjets. There are many investigations focusing on this configuration [2-12]. However, injecting liquid in the form of spray appears more promising in terms of providing better control over trajectory, droplet size and velocity [13-16]. The experimental investigation by Leong et al [13] appears to be the first systematic study of spray injected into a crossflow aimed for gas turbine combustors. Spray trajectory and droplet size and velocity distribution were reported. They have used ambient temperature conditions for all their experiments. Sinha et al [14] built upon their findings and carried out experimental investigation for a much wider range of parameters and using different liquids. They have derived a generalized spray trajectory correlation, which is found to be in good agreement with their experimental results obtained for water and ethanol. Their correlation is also in good agreement with the results of Leong et al [13]. This configuration is also studied numerically and found to be in good agreement with the experimental results [15]. Some recent studies also carried out experiments in high

*For correspondence temperature and/or high-pressure environments [16, 17]. Droplet evaporation is also studied for a spray in crossflow configuration [18].

\subsection{Under-expanded jets}

Nozzles which are at a higher pressure than the ambient are known to form under-expanded jets [19]. Further, underexpanded jets can be divided into two regimes - moderate and strong. For moderately under-expanded gas jets, shockcells or shock-diamonds are formed; while for highly under-expanded jets, formation of Mach discs is also observed [20]. Particle laden under-expanded gas jets are experimentally investigated by Sommerfeld [21, 22]. He observed the presence of similar structures as present in under-expanded gas jets. However, the Mach disc location is found to move upstream with increase in particle loading. Sommerfeld and Kurien [23] also carried out studies for under-expanded jets with droplets and reported the presence of Mach disc and related features. Recently, Buchmann et al [24] have also studied particle-laden underexpanded gas jets and measured particle velocities employing a novel measurement technique. Kihm et al $[25,26]$ and Park et al [27] have studied spray formed by airblast atomizers and investigated the effect of under-expanded jets formed by airblast gas on droplet breakup. Hence, based on the observation in these studies, it can be assumed that the under-expanded gas jet behavior is also exhibited in particle-laden and droplet-laden gas jets or sprays investigated in the present study. However, these 
studies primarily focus on time-averaged behavior of these jets. Unsteady behavior is discussed in section 1.2.

\subsection{Shock-vortex interaction in under-expanded jets}

Under-expanded jets have coherent structures or organized vortices in the shear layer [28]. The locations of these shear layer vortices are marked in a schematic from Mitchell et al [28] shown in figure 1. As the vortices move downstream, they interact with the Mach disc. Consequently, the Mach

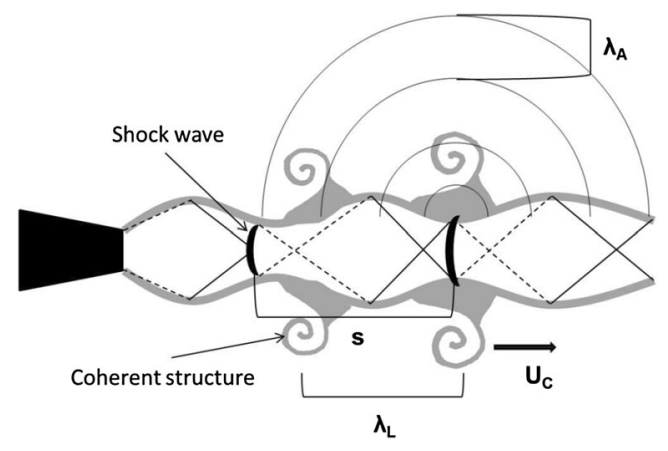

Figure 1. Schematic of under-expanded jet from [28]. The shear layer vortices and Mach disc are shown clearly. The Mach disc is termed as shock wave, and vortices are called coherent structures. disc is observed to oscillate about its mean position. These oscillations are believed to be responsible for the screeching sound produced by these jets. This shock-vortex interaction is studied numerically by Manning and Lele [29] and experimentally by Panda et al [30-32]. Panda and Seasholtz [32] have carried out detailed velocity and pressure measurements to characterize the shock-vortex oscillations and provide some interesting insights into the underlying physics of this configuration. They have also reported the dominant screeching frequency to be around $5400 \mathrm{~Hz}$ for jet with Nozzle Pressure Ratio (NPR) 2.5. This frequency is also found to decrease with increase in NPR. Moreover, the mechanism of this phenomenon can also be understood from the results of Manning and Lele [29]. A sequence of images from their simulations showing the motion of vortex past the shock (shown as thick yellow curve) and shock oscillations is shown in figure 2 .

Note that the pressure pulse believed to be screeching tone is produced at the same instant when the shock disc moves upstream. This transient behavior is also captured in POD modes in [28]. Figure 3 shows a POD mode shape from [28]. This mode shape is obtained from the radial velocity measurements. The conical red and blue features observed in figure 3 are understood to capture the shock oscillations which were captured in figure 2 . To summarize, the under expanded jet has been studied
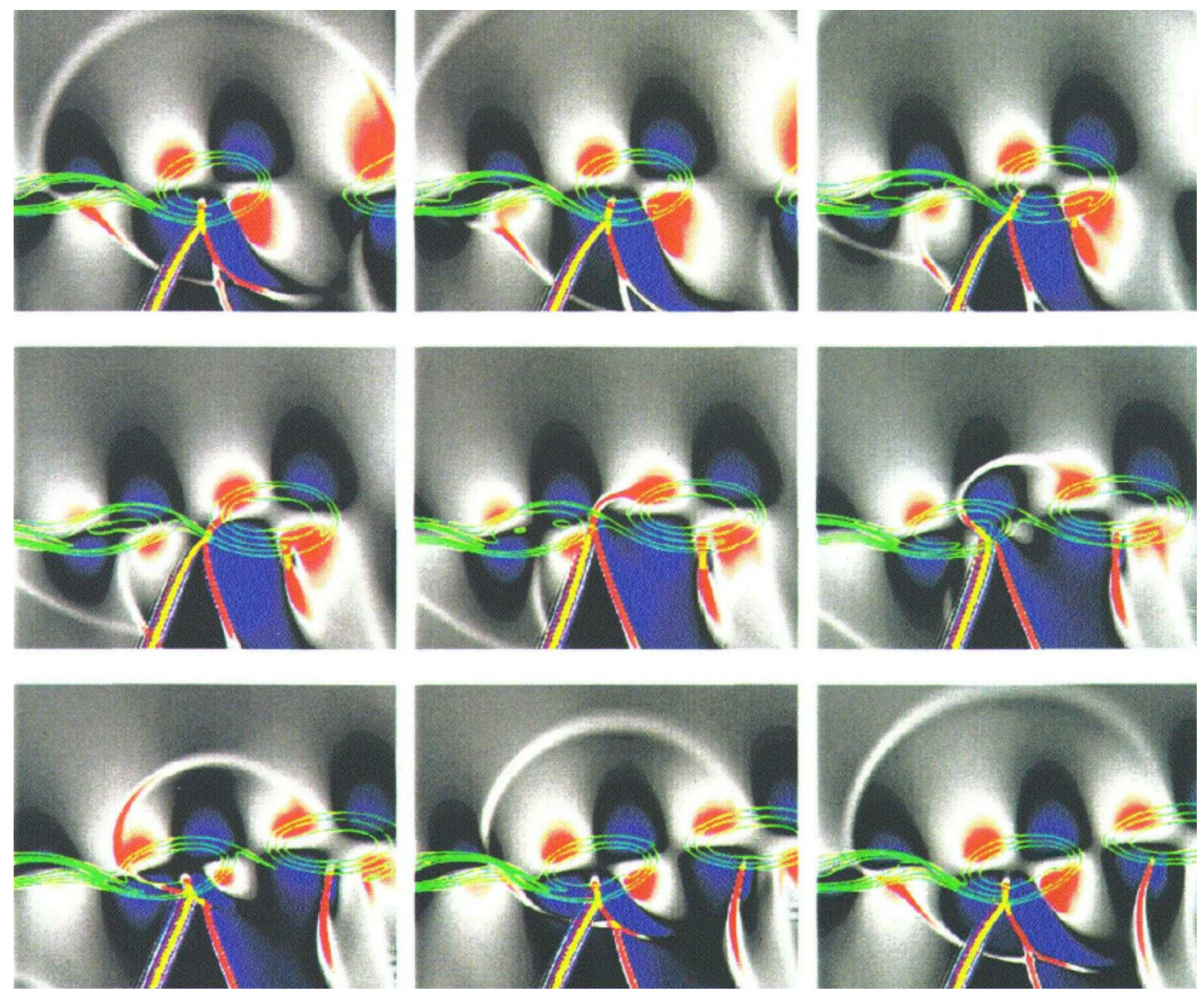

Figure 2. Simulation results from [29] showing the Mach disc oscillation (marked as thick yellow curve) as a vortex moves past it. The pressure waves emanating from the shock edge are believed to be the screeching sound waves. 


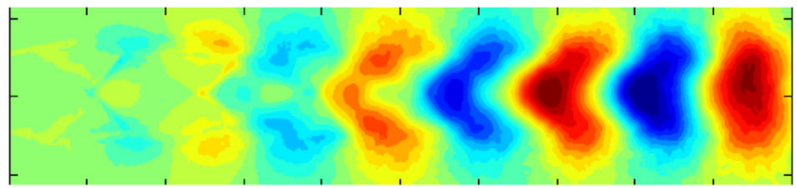

Figure 3. POD Mode shape for under-expanded screeching jet from [28].

in detail, both using experimental and computational tools, but there are very few studies on particle-laden under expanded jets. Further, unsteady behavior of underexpanded gas jets are characterized in detail, but no study is available for particle laden under-expanded jets or spray. As these structures are expected to affect atomization significantly, it becomes important to investigate spray under such conditions. Moreover, these jets also produce a loud screeching tone. The associated frequencies or their sub-harmonics of these tones have potential to get coupled with pressure or heat release

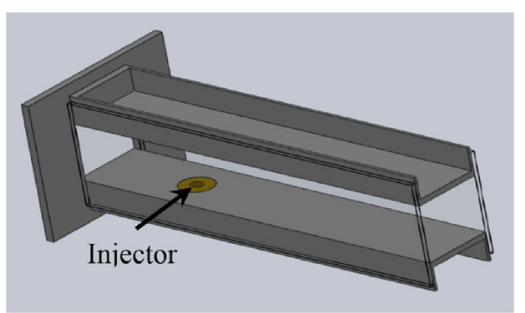

(a)

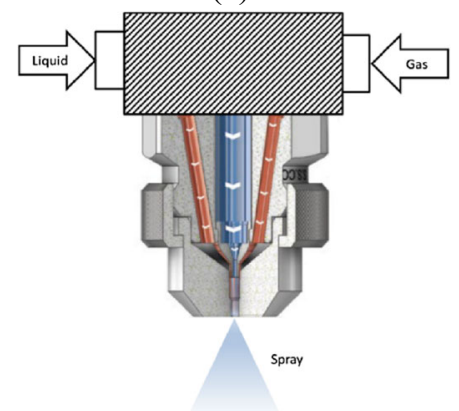

(b)

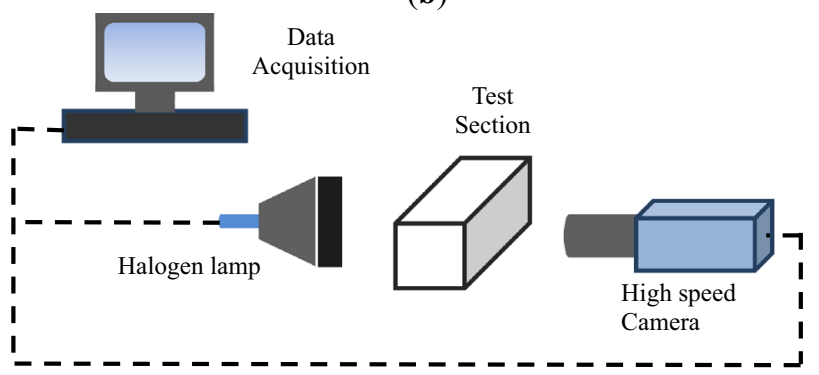

(c)

Figure 4. (a) Schematic of test section, (b) schematic of atomizer internal structure. Liquid line is marked with blue and gas passages are marked with red color. Image adapted from Spraying Systems catalogue [33]. (c) Schematic of imaging facility. oscillations in a combustor and further lead to combustion instability. Hence, a detailed characterization for these behaviors in airblast spray is required. The present study attempts to address this issue. Spray from an airblast atomizer is investigated in detail using high-speed imaging, droplet size measurements and Proper Orthogonal Decomposition (POD) of spray images. This study also attempts to characterize spray behavior for different operating conditions and propose a regime map. Section 2 presents details of the experimental set-up. Results are discussed in section 3. Concluding remarks and summary of results are presented in section 4 .

\section{Experimental set-up}

The experimental set-up consists of an air supply facility for the crossflow air, and liquid and gas supply system for the airblast atomizer. Schematic of the test section is shown in figure 4. The crossflow air is made uniform and the velocity and turbulence profiles are measured and reported elsewhere, along with other details of the experimental facility [14], and only the test section and atomizer will be described here briefly. The test section consists of metal plates for top and bottom wall, and quartz plates from the side walls. It is a rectangular duct of height $50 \mathrm{~mm}$, width $54 \mathrm{~mm}$ and length $250 \mathrm{~mm}$. The atomizer is flush fitted to the center of the bottom plate.

The atomizer used in this study is of internal-mixing airblast type (Make: Spraying Systems, Model: SU11). The

Table 1. Range of experimental conditions used in this study.

\begin{tabular}{lc}
\hline Parameters & Values \\
\hline Crossflow air velocity $(\mathrm{m} / \mathrm{s})$ & $46-103$ \\
Liquid flow rate $(\mathrm{g} / \mathrm{s})$ & $1-4$ \\
Gas-to-liquid $(\mathrm{GLR})$ ratio & $0.1-0.5$ \\
Nozzle Pressure ratio $(\mathrm{NPR})$ & $1.3-9.8$ \\
Momentum flux ratio $\left(\mathrm{q}_{2}\right)$ & $2.4-56.1$ \\
\hline
\end{tabular}

Table 2. Experimental conditions used for Cases 1-5.

\begin{tabular}{lccccc}
\hline Parameters & Case 1 & Case 2 & Case 3 & Case 4 & Case 5 \\
\hline $\begin{array}{c}\text { Crossflow air } \\
\text { velocity (m/s) }\end{array}$ & 66 & 66 & 86 & 103 & 86 \\
$\begin{array}{c}\text { Liquid flow rate } \\
\quad(\mathrm{g} / \mathrm{s})\end{array}$ & 3 & 3 & 2 & 4 & 2 \\
$\begin{array}{c}\text { Airblast gas } \\
\text { flow rate (g/s) }\end{array}$ & 0.3 & 0.9 & 1 & 0.8 & 0.8 \\
$\begin{array}{c}\text { Gas-to-liquid } \\
(\mathrm{GLR}) \text { ratio }\end{array}$ & 0.1 & 0.3 & 0.5 & 0.2 & 0.4 \\
$\begin{array}{c}\text { Nozzle Pressure } \\
\text { ratio (NPR) }\end{array}$ & 2.9 & 7.5 & 8.3 & 6.7 & 6.7 \\
$\begin{array}{c}\text { Momentum flux } \\
\text { ratio (q })\end{array}$ & 11.7 & 34.9 & 36.2 & 13.9 & 15.8 \\
\hline & & & & & \\
\hline
\end{tabular}




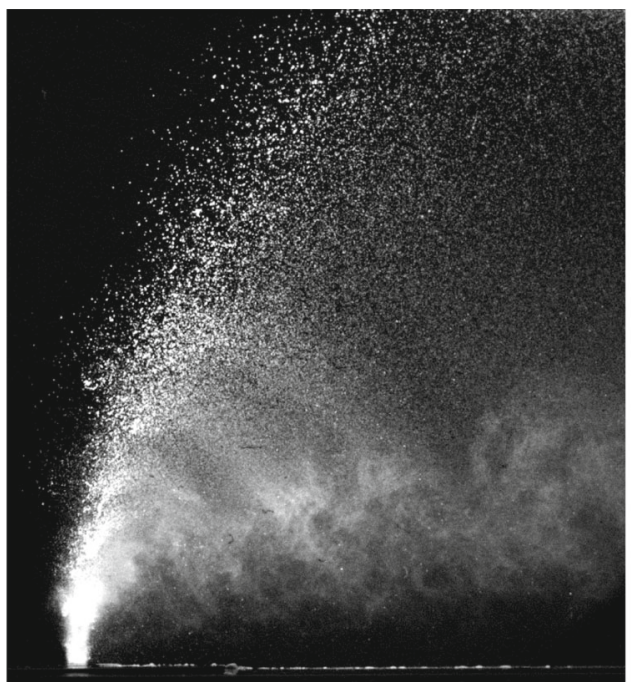

(a) Case 1

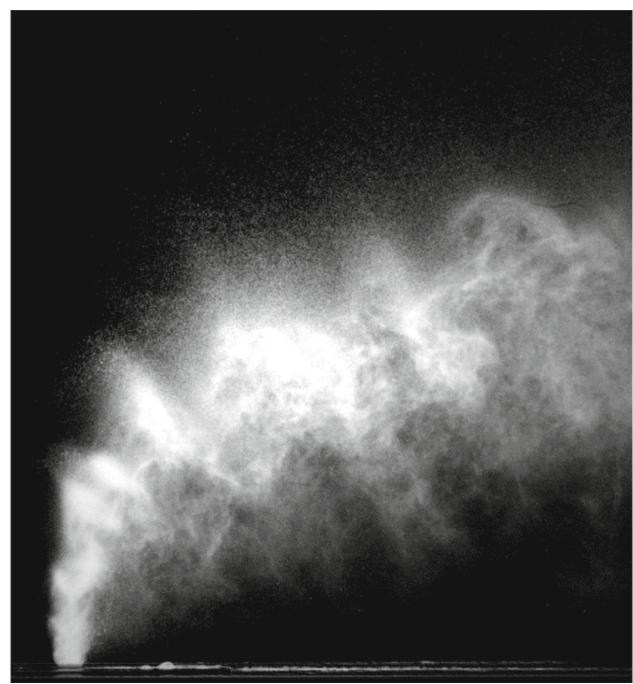

(b) Case 2

Figure 5. Instantaneous images of spray formed in Case 1 and 2 . The images span over an area of $50 \mathrm{~mm} \times 50 \mathrm{~mm}$.

atomizer has two supply lines, one for airblast gas and other for liquid. A schematic of internal structure of the atomizer is given in figure $4(\mathrm{~b})$. Liquid flow rate to the atomizer is controlled by a Coriolis-based mass flow controller (Make: Bronkhorst, Model: mini Cori-Flow). Liquid flow rate is metered within an accuracy of $\pm 0.2 \%$ of flow rate. For airblast gas, nitrogen is filled into a pressure vessel. A pressure regulator and mass flow meter are attached to the outlet of this vessel. The outlet line is finally connected to the atomizer inlet. The flow rate is controlled by adjusting the outlet pressure through the regulator and monitoring it through the gas flow meter. Airblast gas flow rate is measured by Aalborg make mass flow meter (Model: GFM37AVADL2-A0), with an accuracy of $\pm 1.5 \%$ of flow rate. Gas pressure measured at the atomizer inlet is used to calculate NPR. After entering the atomizer, the airblast gas is internally divided into four gas jets (shown in red color in figure 4(b)). The liquid enters the mixing chamber as a single jet (shown in blue color in figure 4(b)). The gas jets impinge on the central liquid jet in the mixing chamber and disintegrate the liquid jet into smaller ligaments and droplets. This mixture of droplets, ligaments and airblast gas exits the atomizer orifice which is $1.2 \mathrm{~mm}$ in diameter.

Pulsed laser with a synchronized camera is used for laser shadowgraphy. A zoom lens is mounted on the camera for spray structure imaging and is used to capture instantaneous spray images with and without crossflow. A long-distance microscope replaced the zoom lens for droplet size measurements using Particle Direct Imaging Analysis (PDIA). The laser imaging system is described in our previous experimental study [14]. For temporally resolved images, a high-speed camera is used. The experimental facility for high speed is shown schematically in figure 4(c). The test section is illuminated by using a $500 \mathrm{~W}$ halogen lamp with a DC supply. A Photron (Fastcam SA5) camera is used, which has the capability to capture images up to a maximum 775000 frames per second. As the field of view and resolution of the camera decreases at the higher frequencies, a frequency of 10000 frames per second is used, where the area is kept limited to near the injector. The area captured in the present study is $25 \mathrm{~mm} \times 25 \mathrm{~mm}$, with a resolution of $0.03 \mathrm{~mm}$ per pixel. The exposure time for this camera is 1 micro-second.

\subsection{Experimental conditions}

Water is used as the test liquid and Nitrogen is used as the airblast gas for all experiments reported in this study. All the experiments are carried out at room temperature and atmospheric ambient pressure for the crossflow air. Liquid and airblast gas in the atomizer are pressurized to obtain airblast spray. Two-phase momentum flux ratio $\left(\mathrm{q}_{2}\right)$ is defined as per Leong et al [14] as:

$$
q_{2}=\frac{\left(\rho_{L} U_{\mathrm{L}}^{2} A_{\text {fuel }}+\rho_{\text {airbl }} U_{\text {airbl }}^{2} A_{\text {airbl }}\right) / A_{\text {spray }}}{\rho_{\text {cross }} U_{\text {cross }}^{2}}
$$

where the subscripts $L$ is for liquid; airbl denotes the airblast gas; and cross stands for the crossflow. The conditions used in this study are summarized in table 1 .

NPR (Nozzle pressure ratio) and GLR (Gas-to-Liquid mass ratio) can be defined as:

$$
\mathrm{NPR}=\frac{\text { Atomizer pressure }}{\text { Ambient pressure }}
$$

GLR $=\frac{\text { Mass flow rate of airbalst gas supplied to atomizer }}{\text { Mass flow rate of liquid supplied to atomizer }}$

Details of experimental conditions for cases which are presented in detail in this study are given in table 2. 


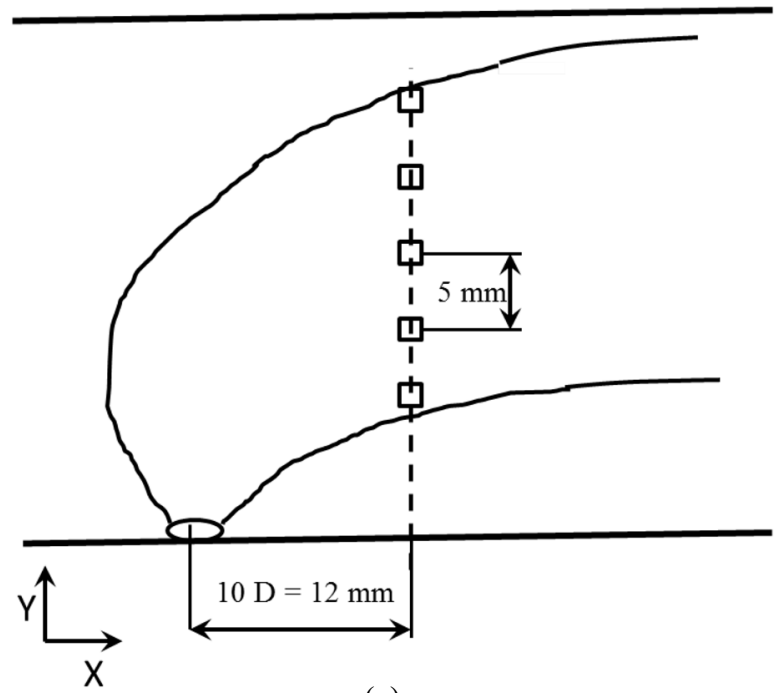

(a)

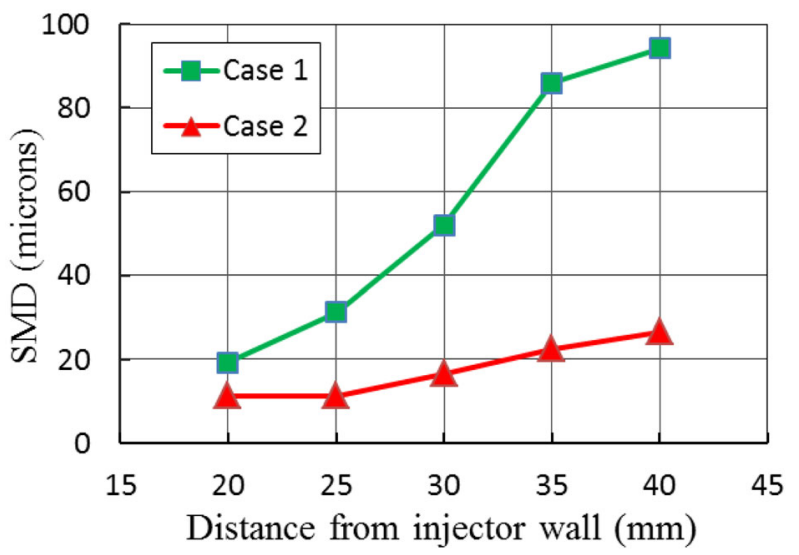

(b)

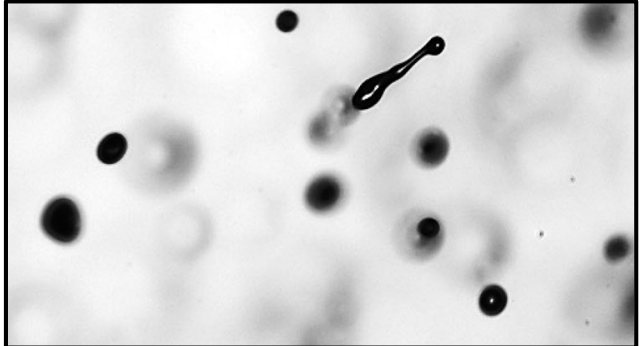

Case 1

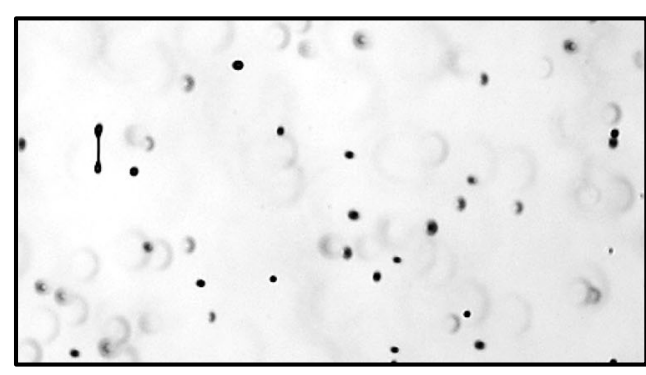

Case 2

(c)

Figure 6. (a) location for drop-size measurements, (b) variation of Sauter Mean Diameter (SMD) with distance from injector wall for Case 1 and 2, (c) typical micrographs used for droplet size measurements.

\section{Results and discussions}

\subsection{Spray in crossflow - effect of underexpanded jet on spray structure}

Typical instantaneous images for two cases are shown in figure 5. Note that both the cases have same crossflow air velocity and liquid flow rate; only the airblast gas flow rate
(GLR) and NPR are different. Still the spray structure is quite different in both the cases. In our previous experimental study [14], it was observed that smaller droplets lose their initial vertical momentum easily and tend to follow the crossflow direction faster as compared to larger droplets, which penetrate the crossflow. Case 1 appears to have larger droplets which penetrate the crossflow while spray in Case 2 appears more dispersed, and having smaller droplets 


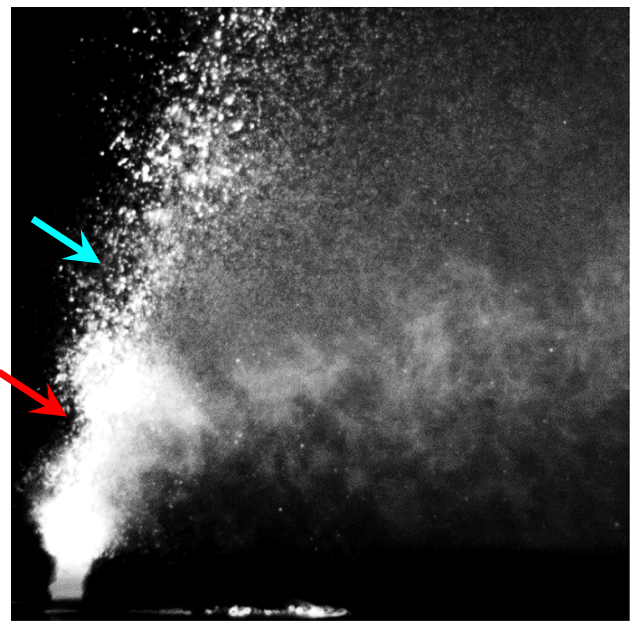

(a) $\mathrm{t}=0$

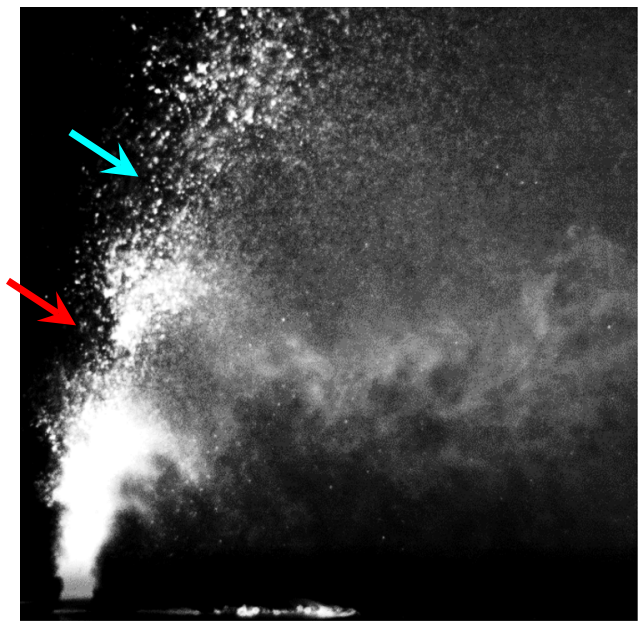

(b) $\mathrm{t}=0.1$ milli seconds $(\mathrm{ms})$

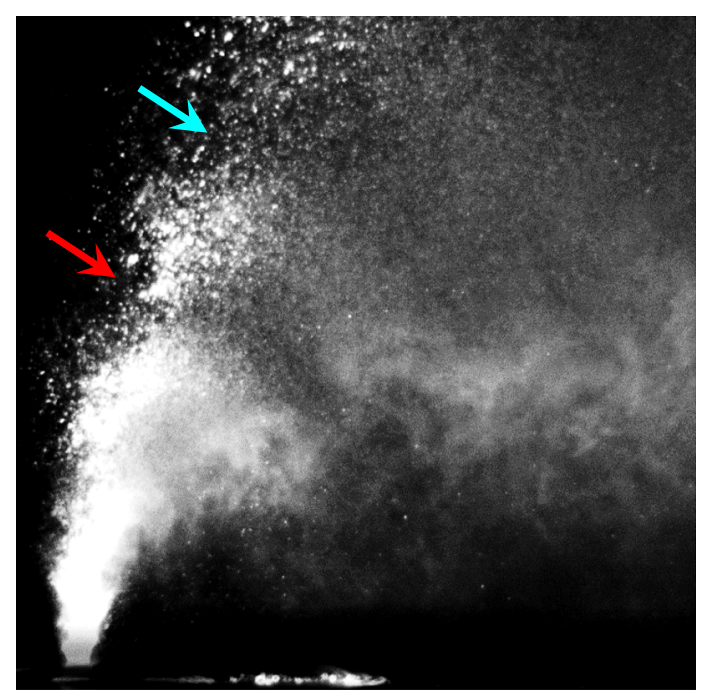

(c) $\mathrm{t}=0.2 \mathrm{~ms}$

Figure 7. Time-resolved instantaneous images for Case 2 . The images span over an area of $25 \mathrm{~mm} \times 25 \mathrm{~mm}$.

which bend easily in the crossflow direction. This is further confirmed by drop-size measurements of these cases. Figure 6(a) shows the locations in crossflow where droplet size measurements have been taken. At a downstream location of 10 nozzle diameters from the injector exit, several vertical locations have been taken for droplet size measurements. Average droplet sizes in terms of Sauter Mean Diameter (SMD) are presented for both the cases in figure 6(b). It is observed that Case 1 has larger droplet size at all the measured locations. Typical droplet micrographs are also shown to compare both the cases in figure 6(c), which again confirm the disparity in droplet sizes. This difference between both these cases can be attributed to the difference in airblast gas which can be expressed in terms of NPR and GLR (see table 2). Regarding the underexpanded jet structure formed by the airblast gas, the two spray cases in the present study can be classified as moderate (Case 1) and highly under-expanded (Case 2) jets owing to their NPRs. Case 2 having an NPR of 7.5 is expected to form a Mach disc.

As discussed in section 1.2, shock-vortex oscillations are observed in under-expanded jets, which are believed to be responsible for noise produced in screeching jets. The spray cases investigated in the present study have airblast gas at a higher pressure than ambient, and hence similar shockvortex interaction can be expected in these spray cases. Capturing Schlieren images of spray structure is not feasible as the droplets block most of the light used for Schlieren imaging, especially in the near-nozzle region. For the present study, spray cases with high NPR have very small droplets which could be assumed to follow the air flow [14] and could be used for flow visualization. High speed images of these spray cases are recorded at 10,000 frames per second to give insight into shock-vortex interaction. 


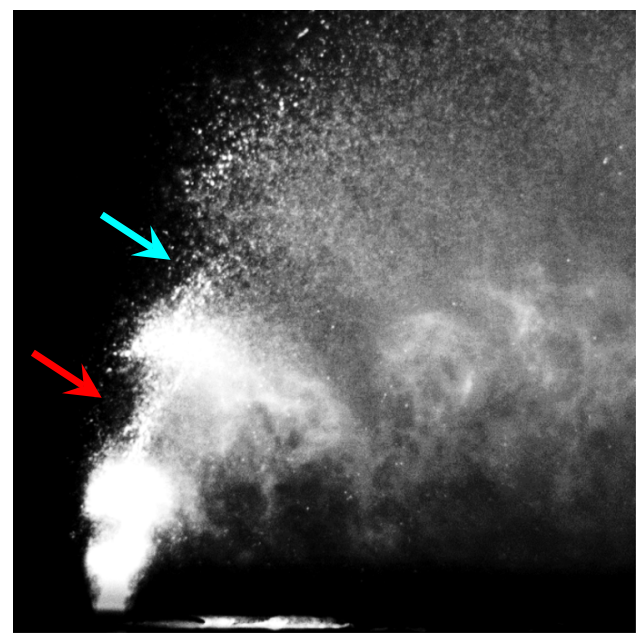

(a) $\mathrm{t}=0$

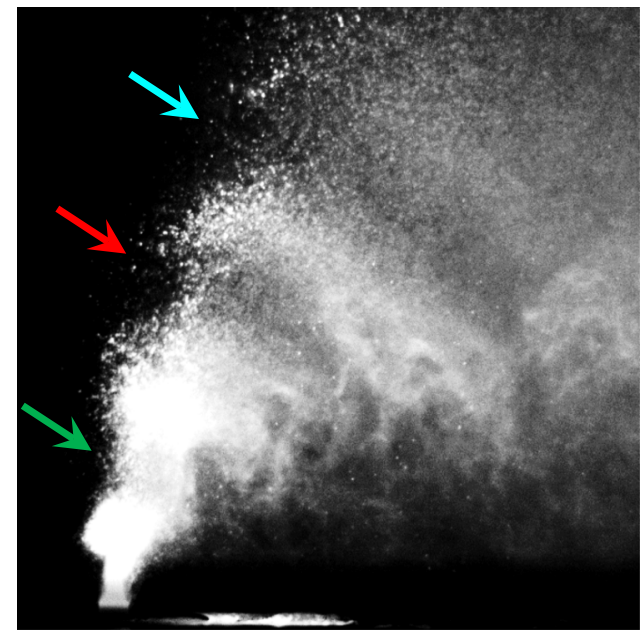

(b) $\mathrm{t}=0.1 \mathrm{~ms}$

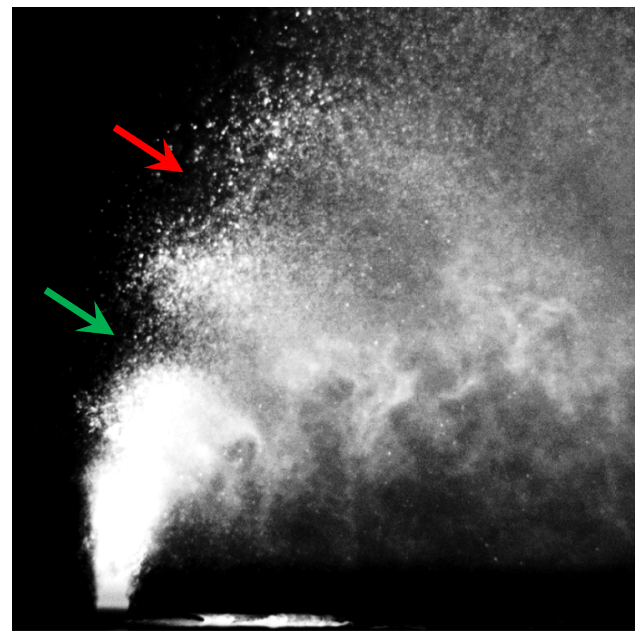

(c) $\mathrm{t}=0.2 \mathrm{~ms}$

Figure 8. Time-resolved instantaneous images for Case 3. The images span over an area of $25 \mathrm{~mm} \times 25 \mathrm{~mm}$.

Typical instantaneous images for Case 2 and Case 3 are shown in figures 7 and 8 . The arrow marks in the images show the location of wave-like patterns which convect downstream along the spray direction. The presence of these patterns can be understood as the following.

It is previously observed that the presence of shock causes droplet sizes to decrease because of higher relative velocity [25]. Now, this relative velocity will further increase while the shock is moving upstream in the oscillation cycle. This upstream motion of the shock is expected to produce much finer droplets which appear as a band like pattern or wave in the instantaneous images of figures 7 and 8 . The droplets are further spread out by the expanding screeching pressure pulse (see figure 1). The motion of this structure with spray closely resembles the behavior observed by Panda for gaseous jets [30-32]. To further investigate this shock-vortex interaction, a Proper Orthogonal Decomposition (POD) study of the spray images is undertaken.

\subsection{Insights from POD study}

POD is a powerful tool used for obtaining low-dimensional approximations of high-dimensional phenomena, by means of identifying the dominant modes or flow features present in a flow field [34-37]. Kosambi [34] was the first to propose the mathematical framework behind POD analysis. Berkooz et al [35] have summarized several applications where POD analysis is found useful. They have also elaborated various mathematical principles that are used in POD analysis. The present study employs snapshot POD, which takes instantaneous images of a flow filed, and processes it to obtain POD modes. These POD modes are mutually orthogonal in space and are arranged in the order of decreasing energy. The basic premise of a POD analysis is that the first $m$ POD modes obtained from a POD analysis are the best $m$ representation of the flow field. Recently, Constantinos [36] has demonstrated the 


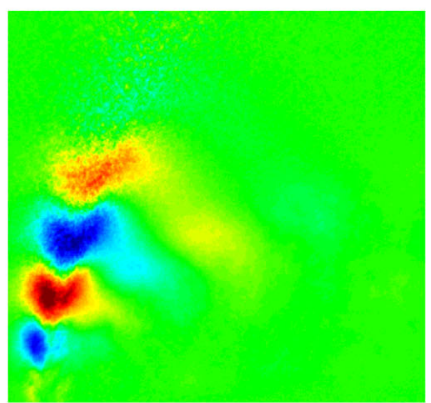

(a) Case 2, mode 2

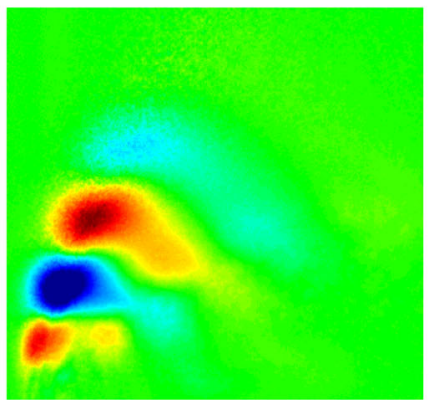

(d) Case 3, mode 2

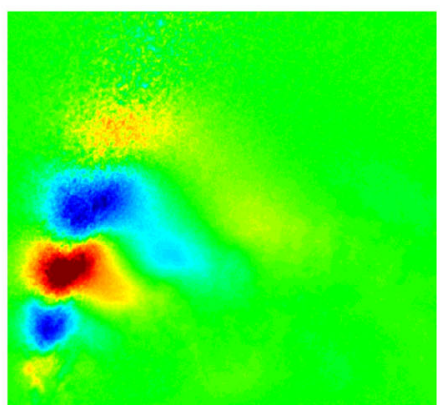

(b) Case 2, mode 3

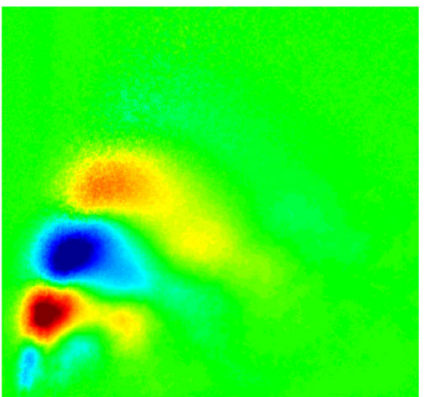

(e) Case 3, mode 3

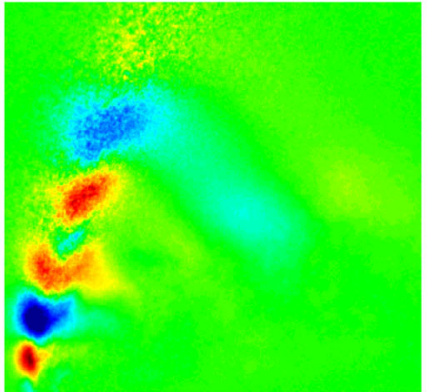

(c) Case 2, mode 4

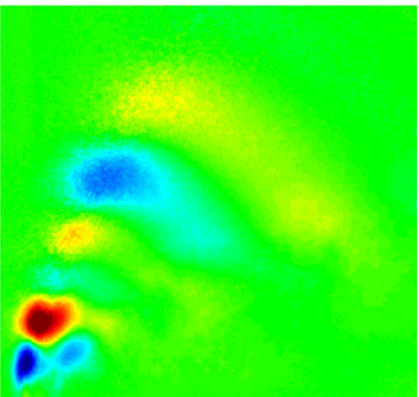

(f) Case 3, mode 4

Figure 9. POD modes for Case 2 and 3.

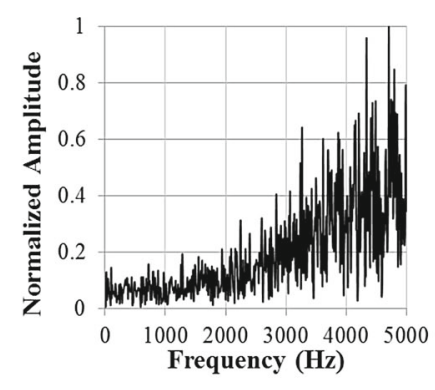

(a) Case 2, mode 2

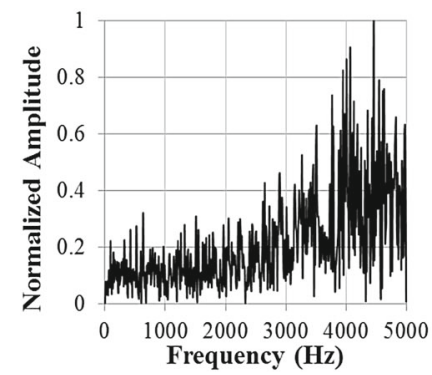

(d) Case 3, mode 2

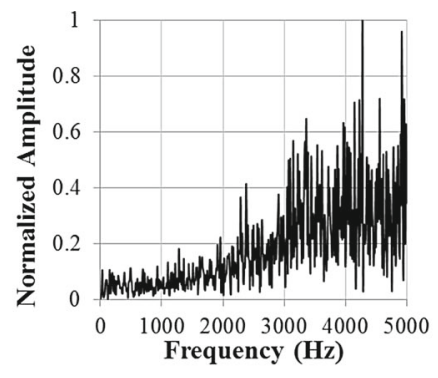

(b) Case 2, mode 3

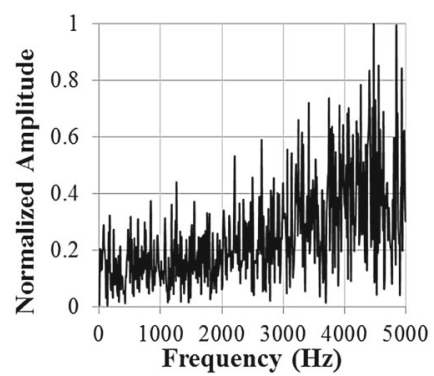

(e) Case 3, mode 3

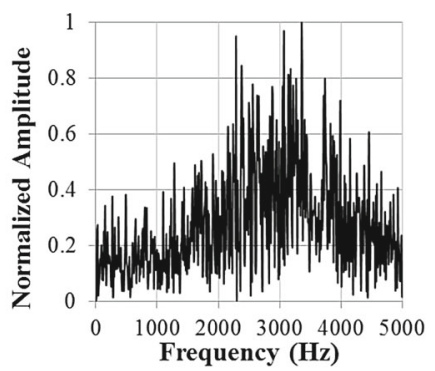

(c) Case 2, mode 4

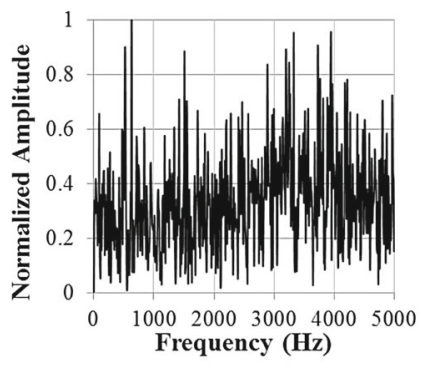

(f) Case 3, mode 4

Figure 10. Power Spectral Density (PSD) for POD modes of Cases 2 and 3.

use of POD analysis in investigating liquid jet breakup and spray formation. In the present study, POD analysis is carried out on the time-resolved spray images obtained by high-speed imaging. The captured droplet locations in the spray are the input to the POD algorithm and any intermittency, such as the behavior observed in figures 7 and 8 can be captured in the POD modes. As discussed previously in section 1.2 , shock-vortex interactions in under- 


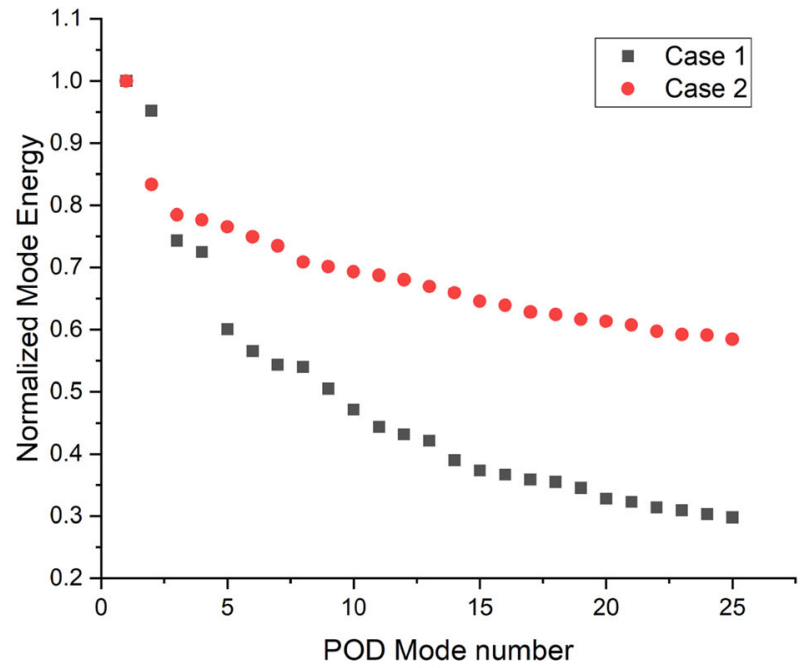

(a)

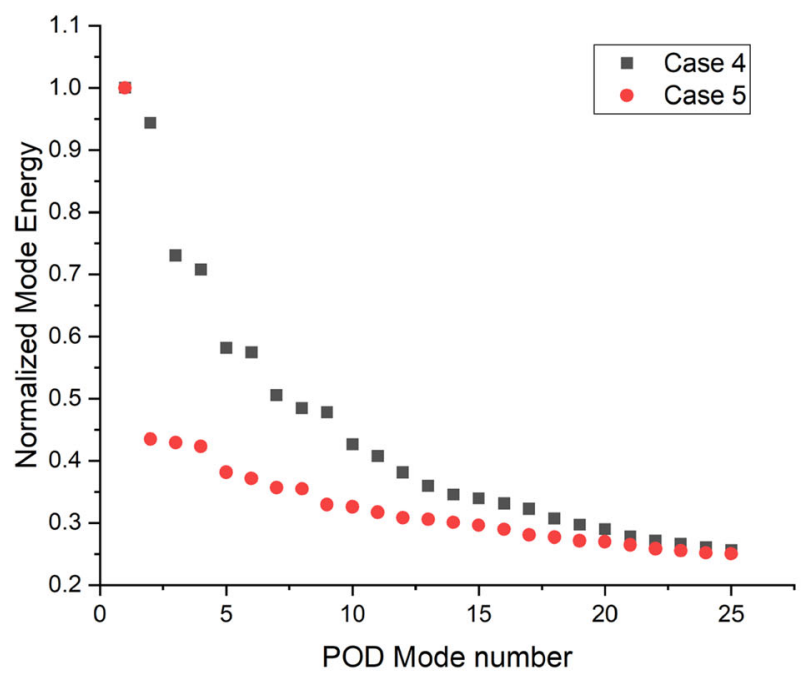

(b)

Figure 11. Normalized mode energies for POD modes.

expanded jets cause shock oscillations. These shockoscillations produce pressure pulses which are believed to generate screeching tones. The measured values of frequency of shock oscillations, pressure pulse and screening tone are all the same, further supporting this hypothesis. It seems the POD mode shapes are able to capture these shock oscillations [28]. The POD mode-shapes for high NPR cases, Case 2 and Case 3 of the present study are shown in figure 9. Mode 1 shows the average spray structure and is not presented here. These mode shapes capture the shock-oscillations. Red and blue nodal structures show alternate locations for shock-oscillations. The similarity between these images and that from [28] (shown in figure 3) is evident, further supporting the hypothesis that spray for high NPR cases shows characteristic features similar to under-expanded gas jets. It should be noted that these oscillations are dying down as the spray penetrates the crossflow. It is expected that at those heights, the crossflow starts dominating, and the under-expanded jet structures seems to dissipate. A similar observation was also made by Panda [30], for jet without crossflow. He mentioned that the shock structures in jet core gradually dissipate and shear layers eventually merge. The corresponding frequencies for these modes are also shown in Power Spectral Density (PSD) of these modes in figure 10. The dominant frequencies also lie in the same range as reported for gaseous under-expanded jets (4000-5000 Hz).

The POD modes are arranged in descending order of their modal energies. Energy here refers to the variance from the mean flow field. Higher the energy, the closer the mode is to the mean spray structure. The energy for first 25 modes is shown in figure 11 for various cases. Energy for Mode 1 is used for normalization for each case. As evident, Cases 2 and 5 have a higher mode 1 and then a steep drop in energy for Mode 2 (specially for Case 5). Thereafter, energy decreases gradually for all other modes. This denotes that the average flow field is most energetic or is more stable for these cases; and the higher modes' stability gradually decreases. Whereas, for Cases 1 and 4, pairs of modes with similar energies are observed. For example, Case 1 shows the following pairs modes 1 and 2, modes 3 and 4, modes 5 and 6 .

High speed images from other cases are also used for POD analysis. Figures 12 and 13 show the POD modes and PSD plots for case 1, 4 and 5, respectively. The differences due to NPR is evident in these images. Case 1 with the lower NPR shows the second mode which resembles the POD of a liquid jet in crossflow [37]. Traces of shockvortex oscillations are also observed in Case 1 for lower modes. But these structures are not as prominent as they are in for Case 2 and 3. This signifies that the high NPR cases (Case 2 and 3) are dominated by the underexpanded jet behavior while for low NPR case, larger liquid droplets and ligaments are dominant. It is also important to note that this dominant mode similar to jet-flapping shows a much lower frequency, around $500 \mathrm{~Hz}$ in contrast to the screeching jet frequencies of around $4000-5000 \mathrm{~Hz}$. This higher frequency is again observed in the PSD for Modes 3 and 4 of Case 1, signifying the weaker energy of shock-vortex oscillations for these cases.

\subsection{Effect of GLR}

Another interesting case is Case 4, which is a high NPR case $(\mathrm{NPR}=6.7)$ but still exhibits liquid jet-like behavior in its first two POD modes. Mode 2 of this case shows a jet-flapping type of behavior which is similar to Case 1 and appears more dominant. Mode 3 also shows similar behavior. The shock-vortex oscillations observed in other cases becomes apparent in Mode 4 of this case. Similarly the PSD frequencies follow the trends discussed above. The jet-like behavior shows a lower 


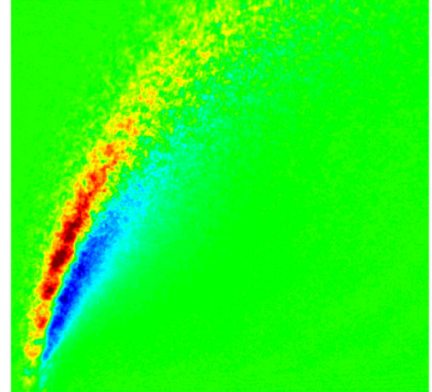

(a) Case 1, mode 2

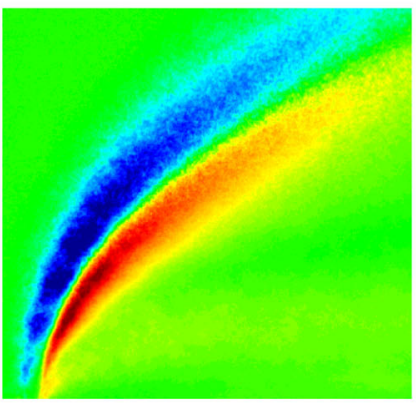

(d) Case 4, mode 2

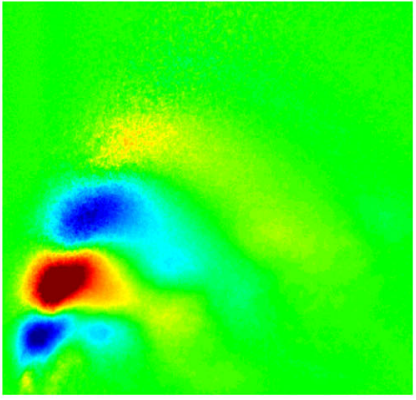

(g) Case 5, mode 2

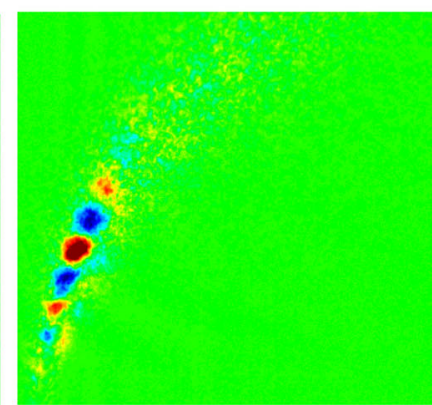

(b) Case 1, mode 3

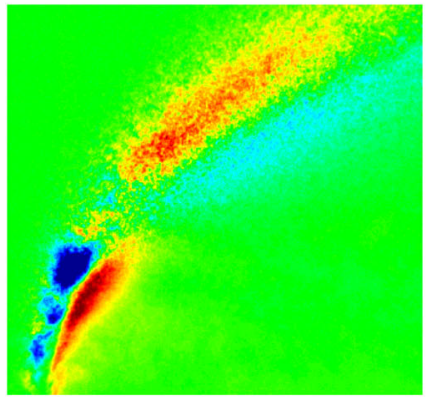

(e) Case 4, mode 3

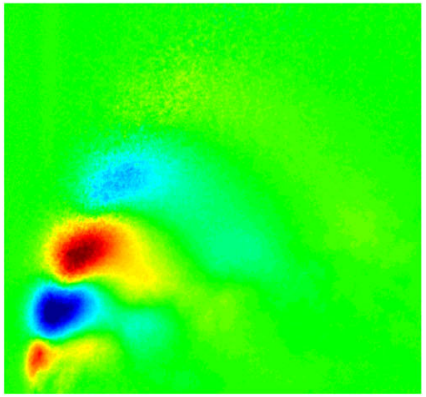

(h) Case 5, mode 3

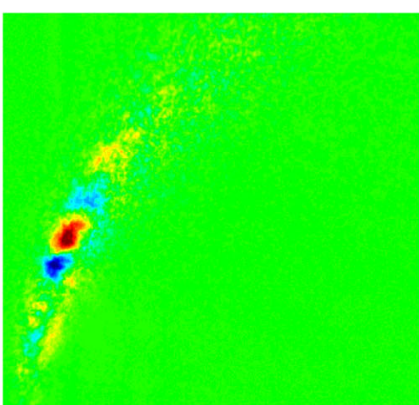

(c) Case 1, mode 4

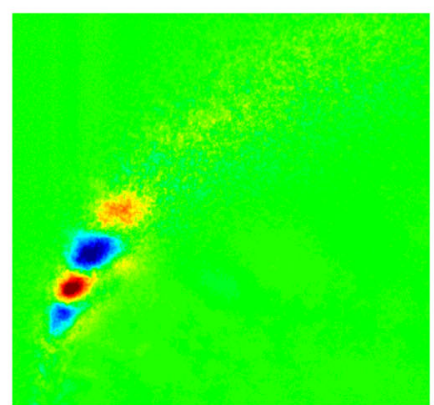

(f) Case 4, mode 4

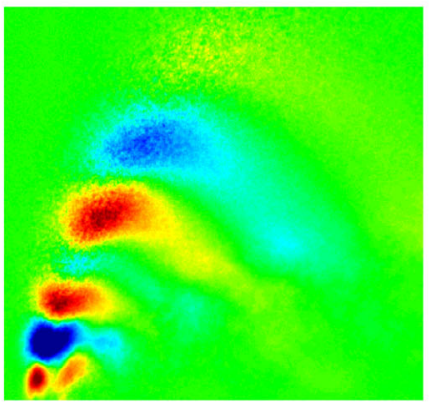

(i) Case 5, mode 4

Figure 12. POD mode shapes for Cases 1, 4 and 5.

frequency, while modes showing the shock-vortex oscillations exhibit a higher frequency of around $3000-4000 \mathrm{~Hz}$. To compare with this case, Case 5 is also investigated, which has the same NPR with this case but a higher Gas-to-Liquid mass ratio (GLR). In contrast to the jet-like behavior shown in Case 4, Case 5 clearly shows signatures of shock-vortex interactions in all three POD modes and high frequency in PSD plots. This apparent difference in behavior of these two cases can be explained as the following. Case 4 is a low GLR case and has larger droplets and ligaments. Also, since the GLR is low, the relative flow rate of gas is much lower than the liquid and hence the droplets and ligaments are not dispersed away from the core where the shock-vortex interaction should be visible. For high GLR and high NPR cases, the droplets are smaller and more dispersed, resulting in the clear visualization of the shock-vortex interaction.
To investigate this further, many other cases with various combinations of GLR and NPR ratios are investigated. A careful analysis of the POD modes, frequency peaks of PSD plots and a large number of high speed images is made. The summary of this study is shown in the form of a Regime map in figure 14. Regime 1 shows the cases in which shock-vortex interaction is the dominant feature. Regime 2 shows the cases which have jet-like flapping as their dominant mode, and Regime 3 is for the intermediate cases which show properties of both the dominant features. As observed, this shock-vortex interaction is clearly visualized for high GLR and high NPR cases and a jet-like flapping is found in cases with low GLR or low NPR. This regime map can also be understood by considering the functioning of the airblast atomizer. The atomizer has two inlets - for liquid and airblast gas (see figure 4(b)). If the gas inlet is closed, and only liquid is supplied, the atomizer produces a liquid jet. Similarly, when the liquid inlet is closed, and 


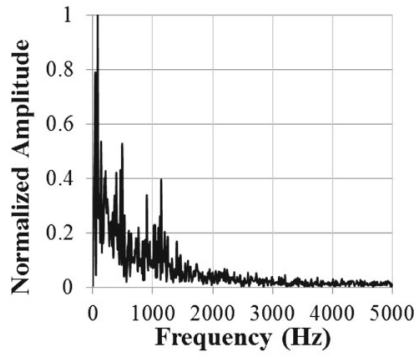

(a) Case 1, mode 2

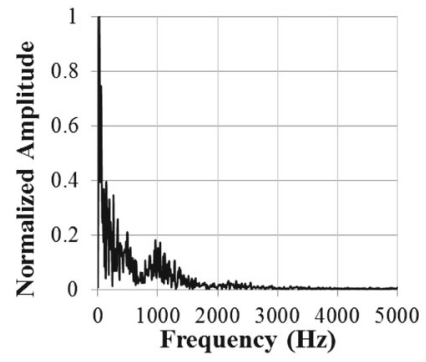

(d) Case 4, mode 2

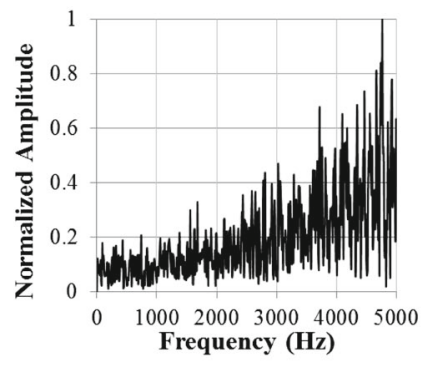

(g) Case 5, mode 2

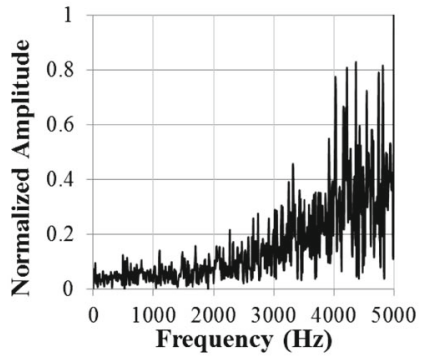

(b) Case 1, mode 3

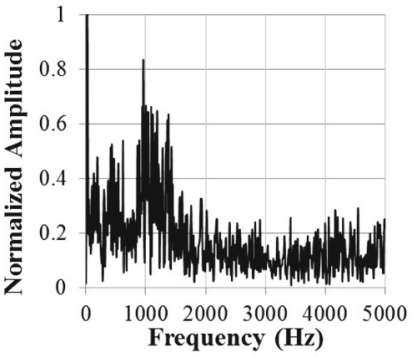

(e) Case 4, mode 3

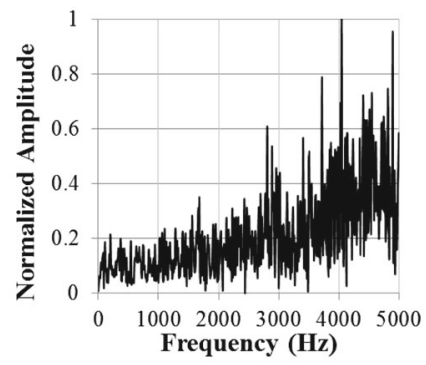

(h) Case 5, mode 3

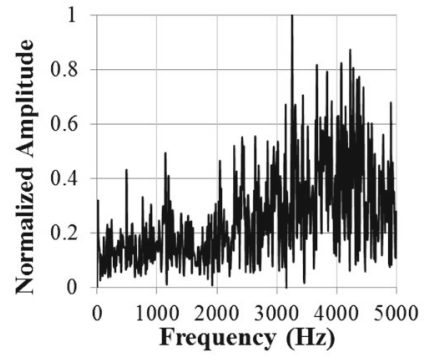

(c) Case 1, mode 4

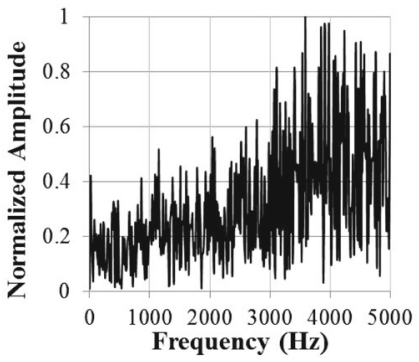

(f) Case 4, mode 4

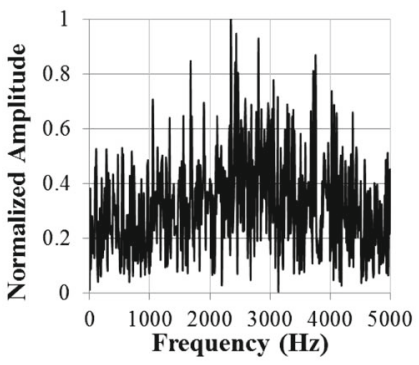

(i) Case 5, mode 4

Figure 13. Power Spectral Density (PSD) for POD modes of Cases 1, 4 and 5.

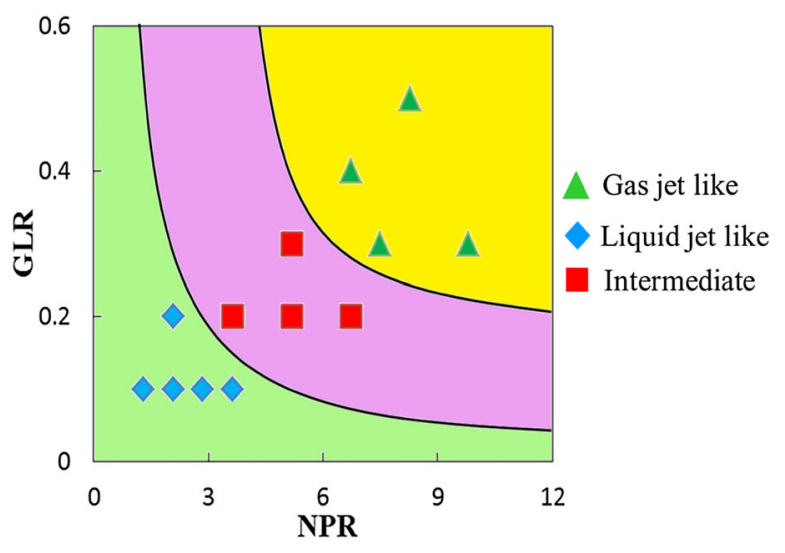

Figure 14. Regime map showing behavior of spray in crossflow.

only gas is provided, the atomizer produces a gas jet. However, when both the inlets are opened, the resulting spray behavior is governed by the dominating phase - liquid or gas. For high NPR and GLR conditions, the gas phase is expected to dominate; and for low NPR and GLR, liquid phase will dominate. This behavior is exhibited in the Regime map in figure 14.

\section{Conclusions}

The structure of an airblast spray in crossflow is investigated using high-speed imaging techniques. A large range of operating conditions is covered, with variation in GLR, NPR, and momentum flux ratio. High speed images of nearnozzle regions show distinct wave-like packets of spray convecting in the downstream direction. POD analysis is performed on these images for different cases. POD modes exhibit shock-vortex oscillations in the near nozzle regions. The dominant frequency of these modes is found to be close to the screech frequency obtained in previous experimental studies. These oscillations are found to be visible for cases with high NPR and GLR. NPR enhances shock strength and 
GLR increases droplet dispersion. Hence, for cases with low NPR and GLR, a flapping liquid jet like behavior is observed. Based on these investigations of spray behavior, a regime map is also proposed.

\section{Acknowledgements}

The authors thank for the financial support provided by the GATET programme of the Aeronautics Research \& Development Board (ARDB), Government of India, for this work. The authors also thank Prof. Satya Chakraborty, and Dr. Ramgopal from IIT Madras for the help in POD analysis used in this study.

\section{References}

[1] Lefebvre A H and Ballal D R 2010 Gas Turbine Combustion 3rd ed., CRC Press

[2] Wu P K, Kirkendall K A, Fuller R P and Nejad A S 1997 Breakup processes of liquid jets in subsonic crossflows. $J$. Propuls. Power 13(1): 64-72

[3] Wu P K, Kirkendall K A, Fuller R P and Nejad A S 1998 Spray structures of liquid jets atomized in subsonic crossflows. J. Propuls. Power 14(2): 173-181

[4] Lee K, Aalburg C, Diez F J, Faeth G and Sallam K A 2007 Primary breakup of turbulent round liquid jets in uniform crossflows. AIAA J. 45(8): 1907-1916

[5] Sallam K A, Aalburg C and Faeth G M 2004 Breakup of round nonturbulent liquid jets in gaseous crossflow. AIAA J. 42(12): 2529-2540

[6] Mazallon J, Dai Z and Faeth G M 1998 Aerodynamic primary breakup at the surface of nonturbulent round liquid jets in crossflow. AIAA Paper 716

[7] Aalburg C, van Leer B, Faeth G M and Sallam K A 2005 Properties of nonturbulent round liquid jets in uniform gaseous cross flows. Atomization Sprays 15: 271-294

[8] Inamura T 2000 Trajectory of a liquid jet traversing subsonic airstreams. J. Propuls. Power 16(1): 155-157

[9] Schetz J A and Padhye A 1977 Penetration and breakup of liquids in subsonic airstreams. AIAA J. 15(10): $1385-1390$

[10] Sinha A, Prakash S R, Raghunandan and Ravikrishna R V 2013 Experimental investigation of jet dispersion in a cross flow. In: National Propulsion Conference, IIT Madras, India, Feb. 21-23

[11] Prakash R S, Sinha A, Raghunandan B N, Tomar G and Ravikrishna R V 2015 Breakup of volatile liquid jet in hot cross flow. Procedia IUTAM, 15: 18-25

[12] Ng C L, Sankarakrishnan R and Sallam K A 2008 Bag breakup of nonturbulent liquid jets in crossflow. Int. J. Multiphase Flow 34: 241-259

[13] Leong M Y, McDonell V C and Samulesen S 2000 Mixing of an airblast atomized fuel spray injected into a crossflow of air, NASA/CR-2000-210467
[14] Sinha A, Surya Prakash R, Madan Mohan A and Ravikrishna R V 2015 Airblast spray in crossflow-structure, trajectory and droplet sizing. Int. J. Multiphase Flows 72: 97-111

[15] Sinha A and Ravikrishna R V 2017 LES of spray in crossflow-effect of droplet distortion. Int. J. Spray. Combust. Dyn. 9(1): 55-70

[16] Lynch A, Batchelor R G, Kiel B, Miller J, Gord J and Reeder M 2011 Spray characteristics of a pressure-swirl fuel injector subjected to a crossflow and a coflow. Atomization Sprays 21: 625-643

[17] Brandt M, Rchner M and Schmitz G 1998 An experimental and numerical study of kerosine spray evaporation in a premix duct for gas turbine combustors at high pressure. Combust. Sci. Technol. 138(1): 313-348

[18] Sinha A, Prakash S, Madan Mohan A and Ravikrishna R V 2016 Experimental studies on evaporation of fuel droplets under forced convection using spray in crossflow methodology. Fuel 164: 374-385

[19] Roshko H W and Liepmann A 2007 Elements of Gasdynamics. Dover Publications

[20] Donaldson C D and Snedeker R S 1971 A study of free jet impingement. Part 1. Mean properties of free and impinging jets. J. Fluid Mech. 45(2): 281-319

[21] Sommerfeld M 1994 The structure of particle-laden, underexpanded free jets. Shock waves 3(4): 299-311

[22] Sommerfeld M 1990 The influence of solid particles on the structure of supersonic free jet flows. In: AIP Conference Proceedings, AIP, vol. 208, No. 1, pp. 745-750

[23] Sommerfeld M and Kurian J 1995 Droplet behaviour in underexpanded supersonic jets. In: Shock Waves at Marseille III. Springer, Berlin, Heidelberg, pp. 95-100

[24] Buchmann N A, Atkinson C and Soria J 2012 Ultra-highspeed tomographic digital holographic velocimetry in supersonic particle-laden jet flows. Meas. Sci. Technol. 24(2): 024005

[25] Kihm K D and Chigier N 1991 Effect of shock waves on liquid atomization of a two-dimensional airblast atomizer. Atomization Sprays 1: 113-136

[26] Kim T K, Son S Y and Kihm K D 1998 Instantaneous and planar visualization of supersonic gas jets and sprays. J. Flow Vis. Image Process. 5: 95-103

[27] Park B K, Lee J and Kimhm D 1996 Comparative study of twin-fluid atomization using sonic or supersonic gas jets. Atomization Sprays 6: 285-304

[28] Edgington-Mitchell D, Oberleithner K, Honnery D R and Soria J 2014 Coherent structure and sound production in the helical mode of a screeching axisymmetric jet. J. Fluid Mech. 748: 822-847

[29] Manning T and Lele S 1998 Numerical simulations of shock-vortex interactions in supersonic jet screech. In: 36th AIAA Aerospace Sciences Meeting and Exhibit, p. 282

[30] Panda J 1998 Shock oscillation in underexpanded screeching jets. J. Fluid Mech. 363: 173-198

[31] Panda J 1999 An experimental investigation of screech noise generation. J. Fluid Mech. 378: 71-96

[32] Panda J and Seasholtz R G 1999 Measurement of shock structure and shock-vortex interaction in underexpanded jets using Rayleigh scattering. Phys. Fluids 11(12): 3761-3777 
[33] Spraying Systems 2018 Automatic and Air Atomizing Spray Nozzles. Product Catalogue, Spraying Systems

[34] Kosambi D D 1943 Statistics in function space. J. Indian Math. Soc. 7: 76-88

[35] Berkooz G, Holmes P and Lumley J L 1993 The proper orthogonal decomposition in the analysis of turbulent flows. Annu. Rev. Fluid Mech. 25: 539-575
[36] Constantinos H 2014 Investigation of break-up process of liquids and downstream spray characteristics in air-blast atomisers. $\mathrm{PhD}$ dissertation, Imperial College, London

[37] Prakash R, Sinha A, Tomar, G and Ravikrishna R V 2018 Liquid jet in crossflow-effect of liquid entry conditions. Exp. Therm. Fluid Sci. 93: 45-56 